\title{
An ovine model of cerebral catheter venography for implantation of an endovascular neural interface
}

\author{
Thomas James Oxley, PhD,,2 Nicholas Lachlan Opie, PhD,,2 Gil Simon Rind, BEng, ${ }^{1,2}$ \\ Kishan Liyanage, MD,1,2 Sam Emmanuel John, PhD, ${ }^{1-3}$ Stephen Ronayne, BEng, ${ }^{1,2}$ \\ Alan James McDonald, ${ }^{2}$ Anthony Dornom, ${ }^{2}$ Timothy John Haynes Lovell, MBBS, ${ }^{1,2}$ \\ Peter John Mitchell, MBBS, ${ }^{4}$ Iwan Bennett, BSc, MBBS, ${ }^{5}$ Sebastien Bauquier, DMV, ${ }^{6}$ \\ Leon Norris Warne, BVMS, DACVAA, ${ }^{6}$ Chris Steward, BSc, ${ }^{4}$ David Bruce Grayden, $\mathrm{PhD},{ }^{3}$ \\ Patricia Desmond, PhD, ${ }^{4}$ Stephen M. Davis, MD, ${ }^{1}$ Terence John O'Brien, MD, ${ }^{1}$ and \\ Clive N. May, PhD²
}

\begin{abstract}
${ }^{1}$ Vascular Bionics Laboratory, Department of Medicine, The Royal Melbourne Hospital; ${ }^{2}$ Neurocardiovascular Laboratory, The Florey Institute of Neuroscience and Mental Health; ${ }^{3}$ NeuroEngineering Laboratory, Department of Electrical \& Electronic Engineering, and Centre for Neural Engineering; ${ }^{4}$ Department of Radiology, The Royal Melbourne Hospital; ${ }^{5}$ Department of Neurosurgery, The Royal Melbourne Hospital; and ${ }^{6}$ Department of Anaesthesia, Veterinary School, The University of Melbourne, Parkville, Victoria, Australia
\end{abstract}

\begin{abstract}
OBJECTIVE Neural interface technology may enable the development of novel therapies to treat neurological conditions, including motor prostheses for spinal cord injury. Intracranial neural interfaces currently require a craniotomy to achieve implantation and may result in chronic tissue inflammation. Novel approaches are required that achieve less invasive implantation methods while maintaining high spatial resolution. An endovascular stent electrode array avoids direct brain trauma and is able to record electrocorticography in local cortical tissue from within the venous vasculature. The motor area in sheep runs in a parasagittal plane immediately adjacent to the superior sagittal sinus (SSS). The authors aimed to develop a sheep model of cerebral venography that would enable validation of an endovascular neural interface.
\end{abstract}

METHODS Cerebral catheter venography was performed in 39 consecutive sheep. Contrast-enhanced MRI of the brain was performed on 13 animals. Multiple telescoping coaxial catheter systems were assessed to determine the largest wide-bore delivery catheter that could be delivered into the anterior SSS. Measurements of SSS diameter and distance from the motor area were taken. The location of the motor area was determined in relation to lateral and superior projections of digital subtraction venography images and confirmed on MRI.

RESULTS The venous pathway from the common jugular vein $(7.4 \mathrm{~mm})$ to the anterior SSS $(1.2 \mathrm{~mm})$ was technically challenging to selectively catheterize. The SSS coursed immediately adjacent to the motor cortex $(<1 \mathrm{~mm})$ for a length of $40 \mathrm{~mm}$, or the anterior half of the SSS. Attempted access with 5-Fr and 6-Fr delivery catheters was associated with longer procedure times and higher complication rates. A 4-Fr catheter (internal lumen diameter $1.1 \mathrm{~mm}$ ) was successful in accessing the SSS in $100 \%$ of cases with no associated complications. Complications included procedure-related venous dissection in two major areas: the torcular herophili, and the anterior formation of the SSS. The bifurcation of the cruciate sulcal veins with the SSS was a reliable predictor of the commencement of the motor area.

CONCLUSIONS The ovine model for cerebral catheter venography has generalizability to the human cerebral venous system in relation to motor cortex location. This novel model may facilitate the development of the novel field of endovascular neural interfaces that may include preclinical investigations for cortical recording applications such as paralysis and epilepsy, as well as other potential applications in neuromodulation.

https://thejns.org/doi/abs/10.3171/2016.11.JNS161754

KEY WORDS sheep; ovine; cerebral venography; catheter; neural interface; brain-machine interface; motor cortex; vascular disorders

ABBREVIATIONS CJV = common jugular vein; CSV = cruciate sulcal vein; ECoG = electrocorticography; ID = inner diameter; IJV = internal jugular vein; IQR = interquartile range; SSS = superior sagittal sinus.

SUBMITTED July 3,2016. ACCEPTED November 7, 2016

INCLUDE WHEN CITING Published online April 28, 2017; DOI: 10.3171/2016.11.JNS161754. 
$\mathrm{I}$ NVASIVE brain-machine interface systems hold promise for the future treatment of paralysis in conditions such as stroke, spinal cord injury, and limb amputation. Closed-loop brain-machine interfaces consist of a sensor, a signal processor, and an electromechanical appendage such as a robotic limb. The sensor functions to record movement-related electrophysiological activity in motor cortex that can serve as a control signal. The sensor must therefore be implanted within sufficiently close proximity of motor cortex to achieve high-fidelity recordings. Functional electrophysiological activity useful for a motor control signal has been demonstrated with a range of recordings, from single unit potentials ${ }^{8,9}$ to electrocorticography (ECoG). ${ }^{25,26}$ The size of intracranial electrodes that have been used in these applications has ranged from millimeters ${ }^{18}$ to centimeters, ${ }^{25}$ and all have required a craniotomy for implantation.

We recently developed a minimally invasive neural interface suitable for endovascular implantation. The electrode array was mounted on a stent scaffold (stentrode) to achieve electrode-vessel wall apposition while maintaining blood flow. Vascular ECoG can be recorded from within the cerebral venous system, and demonstrates a comparable signal quality to that of an invasive epidural array, with a maximal bandwidth of $190 \mathrm{~Hz} \cdot{ }^{15}$ Dexterous robotic limb control has been demonstrated with a closedloop ECoG system utilizing data within a bandwidth between 40 and $200 \mathrm{~Hz}^{25}$ Stentrode signal quality improved following vessel wall incorporation of the electrodes and remained stable for 6 months. ${ }^{15}$ The recorded signal characteristics of the stentrode and capacity for spatial localization on the motor cortical surface may represent a novel minimally invasive approach to a motor prosthesis.

In this paper we report the large animal (ovine) model developed to investigate the anatomical and morphometric features of the cerebral venous system for delivery of an endovascular device in close proximity to motor cortex. The use of small-brained animal models such as the rat have demonstrated the capacity to control a robotic limb using a motor training paradigm, ${ }^{5}$ but research in this discipline shifted toward use of primates due to closer representation to the human motor cortex, and superior capability in fine motor control training. ${ }^{4,18,19,22}$ Despite achieving primate robotic limb self-feeding using this invasive method, ${ }^{24}$ long-term studies have demonstrated a degradation of signal quality resulting from various factors, including gliosis and parenchymal inflammation. ${ }^{3,13,17}$ There is a need to explore less invasive approaches to delivering neural interfaces capable of high spatial localization to motor cortex.

The somatotopic representation of motor cortex of ungulates, including sheep, projects in an anterior direction along and adjacent to the midline in the medial frontal gyrus. ${ }^{2,12,20}$ This contrasts with the motor homunculus of primates, which projects laterally away from the midline. ${ }^{11,16}$ The midline positioning of motor cortex in ungulates places it directly adjacent to the superior sagittal sinus (SSS), offering an ideal hollow structure in which to deposit a neural interface. The SSS is a major draining venous pathway coursing along the vertex of the brain in the midline, forming just behind the frontal sinus anteriorly to join the torcular herophili posteriorly.
We report on a novel ovine model of cerebral venography that characterizes the anatomy, morphometry, and catheterization techniques to target motor cortex using a minimally invasive surgical technique. To achieve the greatest amount of surface area contact with the motor cortex for ECoG recordings, the venous system was selected as the anatomical target, compared with the arterial system that has significantly smaller caliber vessels on the cortical surface. Furthermore, the risk of stroke was considered to be lower in the venous system than the arterial system. We aimed to develop a catheter system and technique to navigate the irregular anatomy of the cerebral venous system, deliver a wide-bore delivery catheter to the anterior SSS, define the location of motor cortex in relation to the venous bifurcations with the SSS, and define the average diameters of the ovine cerebral veins.

\section{Methods \\ Sheep}

Cerebral venography was performed on 39 consecutive sheep during a period from August 2012 to July 2014, at the Vascular Bionics Catheter Laboratory, Florey Institute of Mental Health and Neuroscience, University of Melbourne. Venography and MRI were performed on animals under approval of the Florey Institute of Neuroscience and Mental Health, Animal Ethics Committee. The first 21 sheep were of mixed species, and the final 18 sheep were Corriedales, sourced from a single farm in Werribee, Victoria, Australia (Table 1). The sheep were acclimatized in the institute for a minimum of 1 week prior to venography.

\section{MRI}

Thirteen brain MR images of only Corriedale sheep were obtained prior to venography, under general anesthesia. Sedation with intramuscular midazolam $(5 \mathrm{mg})$ was followed by induction of anesthesia using thiopental. The animals were intubated. Anesthesia was maintained with isoflurane. A postcontrast fast spoiled gradient echo sequence was acquired in the transverse (axial) plane for the purpose of identifying veins and cerebral sinuses. ${ }^{21}$ Gadodiamide contrast (Omniscan, GE Healthcare) $0.1 \mathrm{mmol} /$ $\mathrm{kg}$ was injected intravenously. Field of view was $180 \times 180$ $\mathrm{mm}$, slice thickness $1 \mathrm{~mm}$, flip angle $20^{\circ}$, and number of excitations was 1 . Images were reconstructed in sagittal and coronal planes for analysis.

TABLE 1. Sheep characteristics

\begin{tabular}{lc}
\hline \multicolumn{1}{c}{ Variable } & Value \\
\hline No. of sheep & 39 \\
\hline Mean age in yrs (range) & $4.3(2.5-5)$ \\
\hline Females (\%) & $33 / 39(85)$ \\
\hline Sheep species & Mixed/Corriedale \\
\hline Mean weight in kg (range) & $52(50-57)$ \\
\hline Median horn to horn distance in cm (IQR) & $7.75(7.1-8.4)$ \\
\hline $\begin{array}{l}\text { Median distance from frontal ridge to occipital } \\
\text { protuberance in cm (IQR) }\end{array}$ & $12(11.5-12.5)$ \\
\hline
\end{tabular}




\section{Stent Electrode Array Fabrication}

Stentrodes were fabricated using a commercially available self-expanding stentriever (Solitaire SAB 20, Covidien) as a scaffold on which to mount the recording electrodes. Between 8 and 12, 750- $\mu \mathrm{m}$-diameter, $50-\mu \mathrm{m}$-thick disk electrodes were laser cut from $99.9 \%$ pure platinum foil (Goodfellow). Using a direct current resistance welder (UB25, Miyachi Unitek), these electrodes were attached to polyimide-insulated, $25-\mu \mathrm{m}$-diameter, $92 \%$ platinum $/ 8 \%$ tungsten wire (Goodfellow) and mounted on the stent strut crosslinks at intervals of approximately $3 \mathrm{~mm}$ using an ultraviolet curable, biocompatible adhesive (Dymax, 1128AM, UV Pacific). The wires were wrapped around the stent head such that the mechanical characteristics of the stent were not compromised before being wound around the stent shaft a length of $40-80 \mathrm{~cm} .{ }^{14}$

\section{Catheter Venography}

Sheep were fully shorn and head size measurements recorded. A general anesthetic was begun with an induction dose of thiopentone $(15 \mathrm{ml} / \mathrm{kg})$ and maintenance with isoflurane $(2.0 \%-2.5 \%)$. A 9 -mm endotracheal tube was inserted and mechanical ventilation commenced.

A surgical cut-down of the neck was performed to expose the common jugular vein (CJV), at a point one-third of the distance from the angle of the mandible to the clavicle (Fig. 1). A purse-string suture was placed in the CJV through which the stab was performed with an 18-gauge needle. A nontraumatic $\mathrm{J}$ wire $(0.035$-inch inner diameter [ID], Safe-T-J Curved, Cook Medical) was advanced into the CJV, over which a sheath (0.088-inch ID, NeuronMax, Penumbra) was introduced and secured. An intravenous heparin bolus of 150 units $/ \mathrm{kg}$ was given after the puncture. Activated clotting time was measured at half-hourly intervals (ACT, Medtronic) and maintained above 250 seconds. A mobile C-arm (Arcadis Avantic, Siemens) was used to conduct digital subtraction venography and roadmapping of the venous system during catheterization with injection of a contrast agent (Omnipaque 350, GE Healthcare) diluted 50:50 with normal saline.

Multiple coaxial catheter systems were trialed to determine the widest-bore catheter that could be introduced into the anterior portion of the SSS for potential delivery of a device. We tested delivery success of an incremental size range of catheters into the SSS, including a 6-Fr (0.070-inch ID, Neuron, Penumbra), a 5-Fr (0.059-inch ID, Chaperon 5F outer catheter, MicroVention), and two 4-Fr catheters (0.044-inch ID, DAC, Concentric Stryker, and 0.041-inch ID, Reperfusion; see Table 2 for coaxial catheter system details).

Three significant venous divisions/convergences required coaxial catheter telescoping navigation. First, the convergence of the CJV with the internal jugular vein (IJV), usually occurring at the level of the angle of the

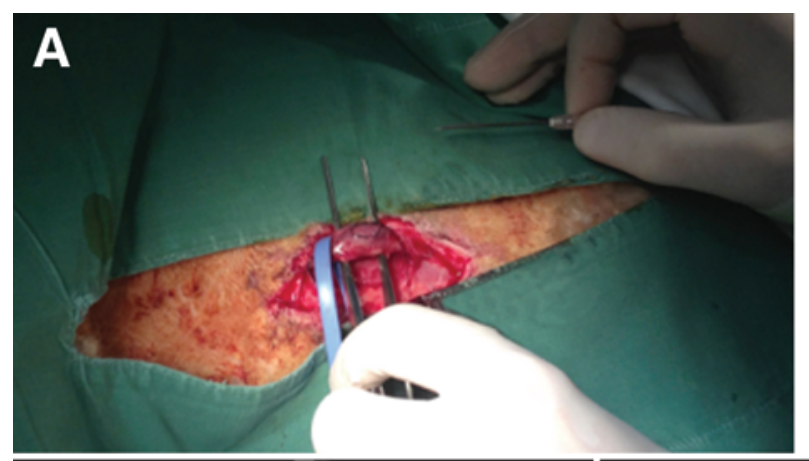

B
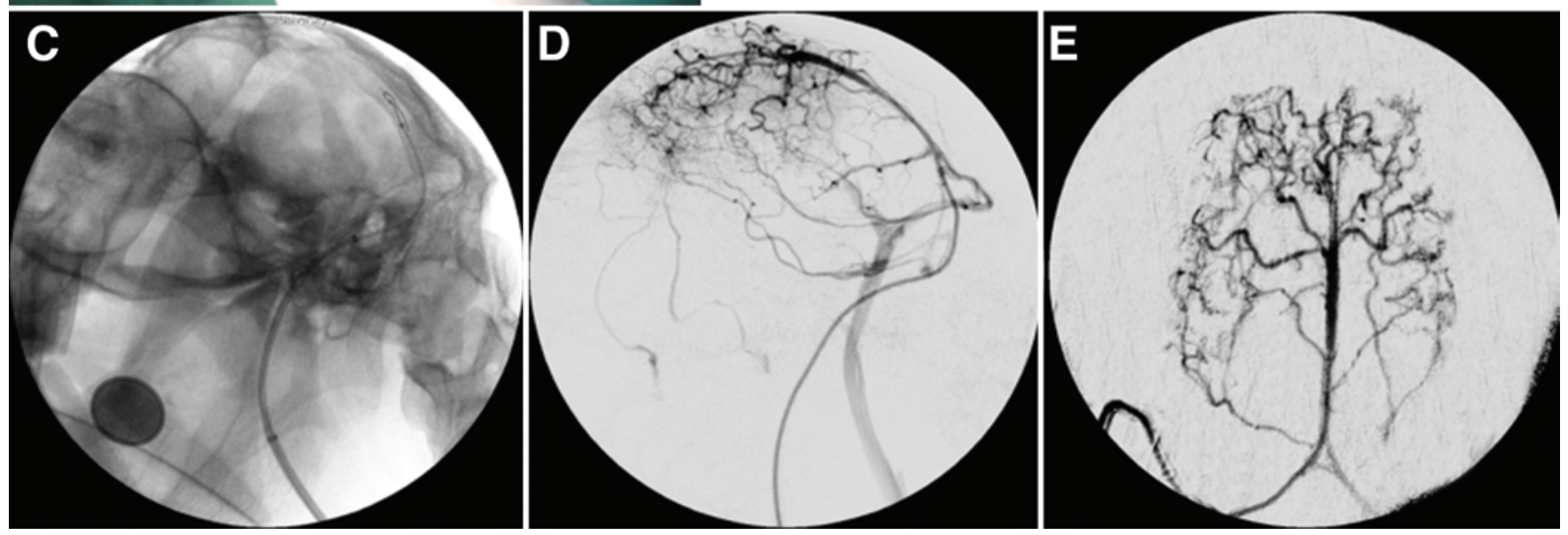

FIG. 1. Ovine cerebral venography. A telescoping coaxial catheter system comprising a sheath and up to 3 catheters was fed over a microwire (B) to navigate the tortuous venous pathway from CJV to SSS. The CJV was exposed via cut-down at the proximal third of the neck (A). Standardized lateral (D) and superior (E) digital subtraction venogram projections were recorded in each animal to record vessel diameters and conduct surgical planning. A native radiograph (C) demonstrates the telescoping catheter system in situ with "J" curve on the leading microwire to reduce trauma. Figure is available in color online only. 
TABLE 2. Comparison of 4 coaxial catheter systems

\begin{tabular}{|c|c|c|c|c|}
\hline \multirow[b]{2}{*}{ System Part } & \multicolumn{4}{|c|}{ Coaxial Catheter System } \\
\hline & 1 & 2 & 3 & 4 \\
\hline \multicolumn{5}{|c|}{ Intended device delivery catheter } \\
\hline Size & $6-\mathrm{Fr}$ & $5-\mathrm{Fr}$ & $4-\mathrm{Fr}$ & $4-\mathrm{Fr}$ \\
\hline ID (in/mm) & $0.070 / 1.7$ & $0.059 / 1.5(1)$ or $0.057 / 1.4(2)$ & $0.044 / 1.1$ & $0.041 / 1.04(1)$ or $0.041 / 1.0(2)$ \\
\hline Name & Neuron & Chaperon (1) or DAC (2) & DAC & Reperfusion (1) or Chaperon (5F Inner) (2) \\
\hline Manufacturer & Penumbra & $\begin{array}{l}\text { MicroVention (1) or Concen- } \\
\text { tric Stryker NV (2) }\end{array}$ & Concentric Stryker NV & Penumbra (1) or MicroVention (2) \\
\hline \multicolumn{5}{|l|}{ Sheath } \\
\hline Size & $7-\mathrm{Fr}$ & $7-\mathrm{Fr}$ & $7-\mathrm{Fr}$ & $7-\mathrm{Fr}$ \\
\hline ID (in/mm) & $0.088 / 2.4$ & $0.088 / 2.4$ & $0.088 / 2.4$ & $0.088 / 2.4$ \\
\hline Name & NeuronMax & NeuronMax & NeuronMax & NeuronMax \\
\hline Manufacturer & Penumbra & Penumbra & Penumbra & Penumbra \\
\hline \multicolumn{5}{|l|}{ Guide catheter } \\
\hline Size & $6-\mathrm{Fr}$ & $5-\mathrm{Fr}$ & $6-\mathrm{Fr}$ & $5-\mathrm{Fr}$ \\
\hline ID (in/mm) & $0.070 / 1.7$ & $0.059 / 1.5(1)$ or $0.057 / 1.4(2)$ & $0.070 / 1.7$ & $0.059 / 1.5$ \\
\hline Name & Neuron & Chaperon (1) or DAC & Neuron & Chaperon \\
\hline Manufacturer & Penumbra & $\begin{array}{l}\text { MicroVention (1) or Concen- } \\
\text { tric Stryker NV (2) }\end{array}$ & Penumbra & MicroVention \\
\hline \multicolumn{5}{|l|}{ Distal access catheter } \\
\hline Size & $4-\mathrm{Fr}$ & $4-\mathrm{Fr}$ & $4-\mathrm{Fr}$ & $4-\mathrm{Fr}$ \\
\hline ID (in/mm) & $0.041 / 1.04$ & $0.041 / 1.0$ & $0.044 / 1.1$ & $0.041 / 1.04(1)$ or $0.041 / 1.0(2)$ \\
\hline Name & Reperfusion & Chaperon (5F Inner) & DAC & Reperfusion (1) or Chaperon (5F Inner) (2) \\
\hline Manufacturer & Penumbra & MicroVention & Concentric Stryker NV & Penumbra (1) or MicroVention (2) \\
\hline \multicolumn{5}{|l|}{ Microcatheter } \\
\hline Size & $2-\mathrm{Fr}$ & $2-\mathrm{Fr}$ & $2-\mathrm{Fr}$ & $2-\mathrm{Fr}$ \\
\hline ID (in/mm) & $0.022 / 0.57$ & $0.022 / 0.57$ & $0.027 / 0.69$ & $0.022 / 0.57$ \\
\hline Name & Excelsior SL-10 & Excelsior SL-10 & Excelsior XL-27 & Excelsior SL-10 \\
\hline Manufacturer & Stryker NV & Stryker NV & Stryker NV & Stryker NV \\
\hline \multicolumn{5}{|l|}{ Microwire } \\
\hline ID (in/mm) & $0.014 / 0.36$ & $0.014 / 0.36$ & $0.014 / 0.36$ & $0.014 / 0.36$ \\
\hline Name & Transend & Transend & Transend & Transend \\
\hline Manufacturer & Stryker NV & Stryker NV & Stryker NV & Stryker NV \\
\hline
\end{tabular}

Use of each system was compared in terms of length of procedure and complication rate. The first row includes the intended delivery catheter of the system, that is, the catheter intended to be used to deliver a device system into the SSS.

mandible, was easily negotiated with a microcatheter-overmicrowire technique. Second, the convergence of the IJV with the maxillary vein, which enters the skull via the temporal meatus to form the temporal sinus, and then becomes the transverse sinus. ${ }^{10}$ The temporal sinus originates at the confluence of sinuses, or torcular herophili, formed by the convergence of the superior and inferior sagittal sinuses. Navigation of this third and largest convergence and into the SSS was the most challenging aspect of the procedure.

To negotiate catheter access of the torcula, all catheters (including the sheath) were telescoped up the IJV to sit in the sigmoid sinus. A digital subtraction contrast run was performed to create a roadmap of the torcula and SSS. The microwire was passed into the torcula and contacted against the posterior wall to create a $J$ shape in the wire, and advanced forward to enable entry into the SSS. The microcatheter was passed over the microwire, and ad- vanced upwards into the SSS at a $90^{\circ}$ angle, with the Jshaped wire leading as an atraumatic leading edge.

Advancement of the 4-Fr catheter through the torcular herophili required support from the guide $(6-\mathrm{Fr})$ catheter. There was a tendency for the 4-Fr catheter to pressure the back wall and hook downward toward the inferior sinus. The usual solution was to advance the guide catheter (6Fr) into the torcula to support the advancing 4-Fr catheter.

\section{Vein and Sinus Diameters}

Vein diameters were manually measured from the CJV to frontal lobe cortical veins using images from both MRI and digital subtraction venography. Open-source imaging analysis toolkit 3DSlicer (http://www.slicer.org) ${ }^{6}$ was used to tag fiducial points along the course of the SSS. Fiducial points were placed in 5-mm increments, commencing at 

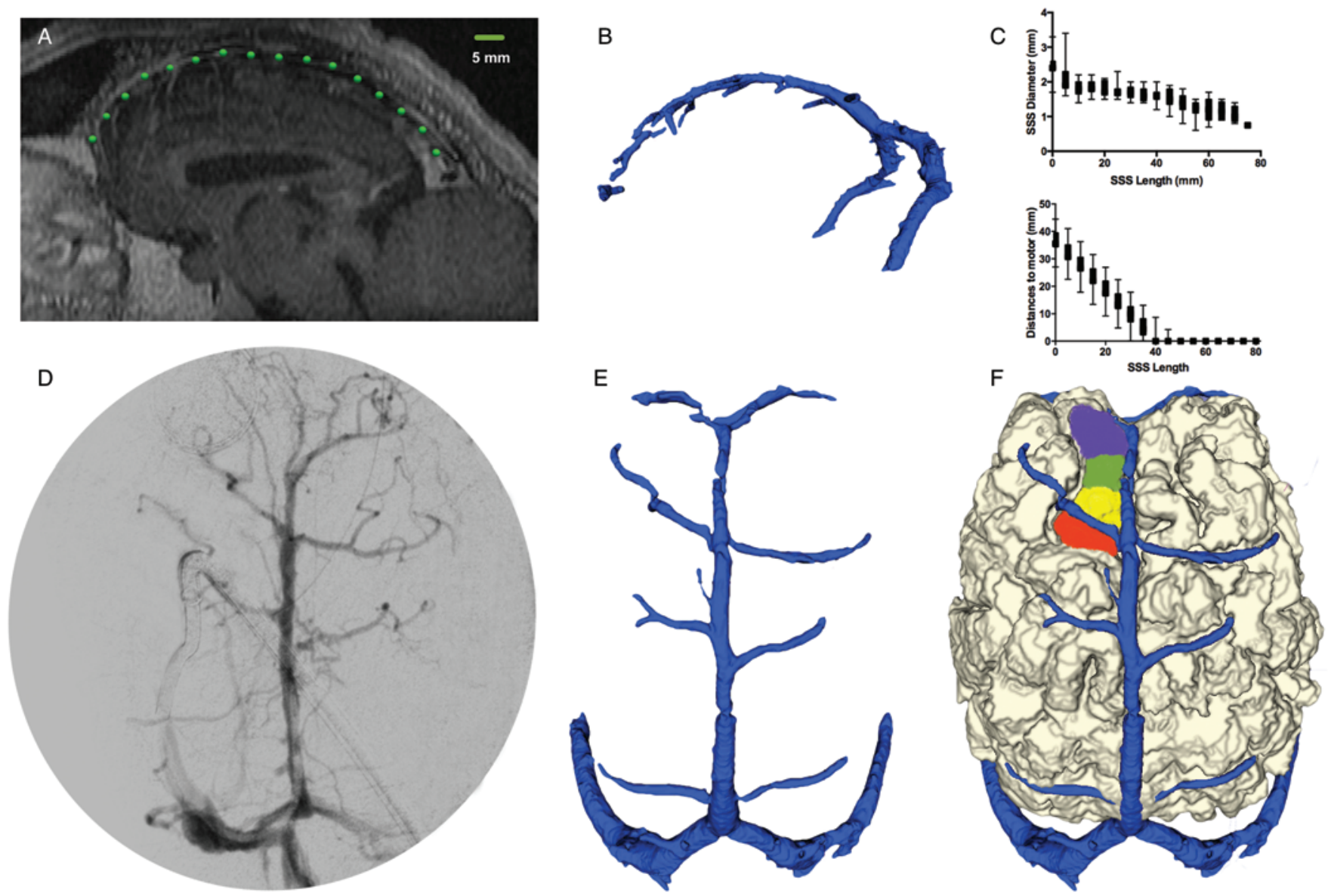

FIG. 2. Sheep cerebral venography in relation to motor cortex. A sagittal plane MRI slice in the midline $(\mathbf{A})$ demonstrates allocated fiducial points (green dots) along the course of the SSS, commencing at the torcular herophili and separated by 5-mm increments. Box and whisker plots (C) demonstrate the SSS diameters (upper) and distance from motor cortical surface (lower) at each incremental fiducial point along the SSS. Three-dimensional reconstructions of the SSS in a lateral (B) and superior (E) projection of 1 animal are shown, with the corresponding superior projection from digital subtraction venography (D). Graphical representation of the motor cortex from cortical stimulation mapping literature ${ }^{2}$ demonstrates somatotopic representation of motor function geographically across the superior frontal gyrus $(F ; r e d=$ hind limb, yellow $=$ forelimb, green $=$ head and eyes, purple $=$ face, mouth, and tongue). Reconstruction using MRI images of the cortical surface of an individual sheep brain with superimposed SSS reconstruction demonstrates motor areas in relation to CSVs $(F)$. Figure is available in color online only.

the bifurcation of the SSS into the transverse sinuses in an anterior direction until the sinus origin of the SSS from anterior cortical veins (Fig. 2). Distances along the SSS at points of convergence with cortical veins were measured to characterize anatomical variability. The SSS was reconstructed using the VMTK module (www.vmtk.org) ${ }^{1}$ in 3D slicer from the contrast-enhanced MR images, for the purpose of visual inspection and surgical planning.

\section{Motor Cortex Distances}

The motor cortex was identified as the superior frontal gyrus based on previous cortical stimulation mapping studies in sheep (Fig. 2).,7,20 Axial contrast-enhanced 1-mm MRI slices were assessed in 3DSlicer to determine the relationship between the location of the SSS and the motor area. Distances in $\mathrm{mm}$ from the nearest cortical surface of the left and right motor area in the superior frontal gyrus was measured at each fiducial point along the course of the SSS and the cortical veins running adjacent to the superior frontal gyrus. The cortical surface of the brain was segmented using ITK-SNAP, ${ }^{27}$ rendered and superimposed on the SSS reconstruction using 3DSlicer (Fig. 2).

\section{Results}

\section{Cerebral Veins and Location to Motor Areas}

The venous puncture was performed in the CJV twothirds up the length of the neck. The median size of the CJV was $7.4 \mathrm{~mm}$ (interquartile range [IQR] $6.2-8.6 \mathrm{~mm}$ ), and that of the IJV was $4.4 \mathrm{~mm}$ (IQR $3.5-6.1 \mathrm{~mm}$ ). The median transverse sinus diameter was $2.5 \mathrm{~mm}$ (IQR 1.9$3.4 \mathrm{~mm}$; Table 3).

Diameters of the SSS are reported at fiducial points separated by increments of $5 \mathrm{~mm}$, commencing at the proximal junction with the torcular herophili. The SSS diameter at the proximal $0-\mathrm{mm}$ fiducial point was $2.4 \mathrm{~mm}$ (IQR 2.4-2.5 mm), reducing in size to $1.2 \mathrm{~mm}$ (IQR 1.0$1.5 \mathrm{~mm}$ ) at the distal 60 -mm fiducial point. The cortical vein of interest was the cruciate sulcal vein (CSV), which bifurcated with the SSS at a median distance of $39.2 \mathrm{~mm}$ 
TABLE 3. Vein and sinus diameters

\begin{tabular}{lc}
\hline \multicolumn{1}{c}{ Vessel } & Median Vessel Diameter in mm (IQR)* \\
\hline CJV & $7.4(6.2-8.6)$ \\
\hline IJV & $4.4(3.5-6.1)$ \\
\hline Transverse sinus & $2.5(1.9-3.4)$ \\
\hline SSS & \\
\hline Proximal (0-mm fiducial) & $2.4(2.4-2.5)$ \\
\hline$\quad$ Middle (30-mm fiducial) & $1.7(1.6-1.8)$ \\
\hline Distal (60-mm fiducial) & $1.2(1.0-1.5)$ \\
\hline CSV & $1.1(1.0-1.2)$ \\
\hline
\end{tabular}

* Blood vessel measurements from cerebral venograms $(n=28)$ and concurrent MRI brain scans with contrast $(n=13)$, to characterize cerebral vein diameters.

(IQR 37.8-41.1 mm) on the left hemisphere and $35.9 \mathrm{~mm}$ (IQR 34.3-38.1 mm) on the right hemisphere. This bifurcation was an accurate indicator of the motor area in the superior frontal gyrus, which commenced at the $40-\mathrm{mm}$ fiducial point (IQR 40-40 mm). The CSV diameter was a median of $1.1 \mathrm{~mm}$ (IQR 1.0-1.2 mm). The left CSV bifurcated with the SSS at a median angle of $43^{\circ}$ (IQR $34^{\circ}-56^{\circ}$ ), while the right bifurcated at a more obtuse angle of $90^{\circ}$ (IQR $22^{\circ}-115^{\circ}$ ).

The 40-mm fiducial point within the SSS represented the position, anterior to which, the cortical motor area lay immediately adjacent to the sinus. The diameter of the sinus at this point was $1.7 \mathrm{~mm}$ (IQR $1.6-1.8 \mathrm{~mm}$ ) and became smaller in the anterior direction.

\section{Catheter Venography}

We report results from 39 procedures over a technical learning period of catheter venography that includes procedure time and complication rate, related to success in gaining access to the SSS using a particular coaxial catheter system. Procedure time was defined as the time from jugular puncture to SSS access with a particular catheter (Table 4). The aim was to determine the widest bore catheter that could be delivered into the SSS for delivery of a stent-based device. Microcatheter (2-Fr) and 4-Fr catheter access into the SSS was achieved in $100 \%$ of cases with no complications. Attempts were made with 6-Fr catheters, which were successfully introduced into the SSS in $9(82 \%)$ of 11 cases, but at the expense of a long procedure time (median 61 minutes, IQR 45-72 minutes) and a significant complication rate. 5-Fr catheters were successfully introduced into the SSS in 13 (72\%) of 18 attempts (median 43 minutes, IQR 30-57 minutes), but also occurring with significant rates of complication.

\section{Complications}

Procedure-related complications occurred almost exclusively with the attempted use of 5-Fr and 6-Fr catheters to access the SSS. Complications were predominantly venous dissection and subdural hemorrhage, but also included acute thrombus formation. The only complication not related to 5-Fr or 6-Fr catheter use included a venous puncture from a guidewire (0.035-inch ID, Benston, Cook Medical) that led to a subdural hemorrhage. Following this
TABLE 4. Rates of success with catheter access into the SSS, complications, and procedure times

\begin{tabular}{lccc}
\hline \multicolumn{1}{c}{ Catheter } & $\begin{array}{c}\text { Success } \\
\text { in SSS } \\
\text { Access (\%) }\end{array}$ & $\begin{array}{c}\text { Complication } \\
\text { Rate (\%) }\end{array}$ & $\begin{array}{c}\text { Median } \\
\text { Procedure Time } \\
\text { in Min (IQR) }\end{array}$ \\
\hline Microcatheter (2-Fr) & $38 / 38(100)$ & 0 & $32(20-41)$ \\
\hline 4-Fr (0.041-in) & $31 / 31(100)$ & 0 & $38(24-45)$ \\
\hline 4-Fr (0.044-in) & $7 / 7(100)$ & 0 & $48(34-63)$ \\
\hline $5-\operatorname{Fr}(0.057 / 0.059-$ in) & $13 / 18(72)$ & $6 / 18(33)$ & $43(30-57)$ \\
\hline $6-\operatorname{Fr}(0.070-$ in) & $9 / 11(82)$ & $4 / 11(36)$ & $61(45-72)$ \\
\hline
\end{tabular}

case, only microwires (0.014-inch ID, Transend 14, Stryker Neurovascular) were used, with no further cases of venous puncture. Use of the 5-Fr catheters was associated with complications in $6(33 \%)$ of 18 cases. All complications were subdural hemorrhages from venous dissection due to catheter manipulation. Four of the 6 dissections occurred with navigation around the torcular (confluence of sinuses) leading to brainstem subdural hemorrhage. The other two occurred with catheterization of anterior cortical veins near the commencement of the SSS (Fig. 3). Of the two 5-Fr wide-bore distal access catheters used, the 5-Fr Chaperon (0.059-inch ID, Chaperon 5-Fr outer catheter, MicroVention Terumo) was more likely to achieve access to the SSS (13/15 attempts) but accounted for 5 of the 6 hemorrhages, due to its comparatively stiffer properties than the 5-Fr DAC (0.057-inch DAC057, Concentric Stryker). Conversely, the softer and more flexible DAC057 only achieved access in 1 of 3 attempts, and was still associated with 1 case of major hemorrhage at the torcular. Five of the 6 hemorrhages led to nonrecovery after general anesthesia. Access to the SSS with use of a 6-Fr catheter (Neuron 070, Penumbra) was also associated with a high complication rate (4 [36\%] of 11). Two of these complications included subdural hemorrhages at the torcular and in the anterior cortical veins, both leading to nonrecovery after anesthesia. The other two complications were due to thrombus formation within the SSS, likely due to a combination of occlusion of the vessel with the catheter as well as longer procedure time. Neither thrombosis complication was related to any clinically detectable morbidity.

\section{Discussion}

We recently reported the first long-term recordings of vascular ECoG in awake animals from a novel endovascular stent electrode array. In this paper we report the novel surgical catheter technique to access the SSS with a widebore delivery catheter as well as define the cerebral venous structures with regard to their proximity to immediately adjacent motor areas in adult Corriedale sheep. This large animal model may facilitate the development of a range of applications of endovascular neural interfaces.

Following early anatomical exploration via craniotomy, we hypothesized that the bifurcation of the CSV was a useful landmark for determination of the commencement of the motor area in the superior frontal gyrus. Evaluation of brains after contrast-enhanced MRI confirmed this hypothesis, with the CSV proving to be an accurate marker 

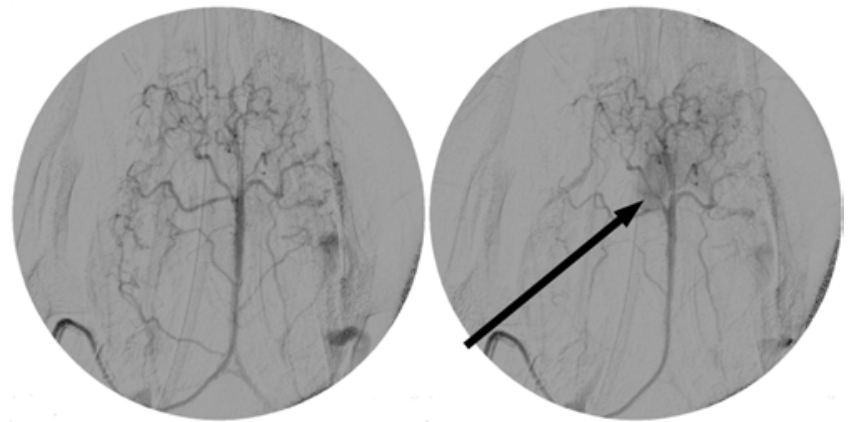

FIG. 3. Example of a complication. Before (left) and after (right) a dissection of the origin of the CSV leading to a subdural hemorrhage (arrow) in the frontal lobe bilaterally, shown on a superior projection.

and converging with the SSS within $4 \mathrm{~mm}$ of commencement of the motor area. Somatotopic motor representation in the sheep has been defined with motor cortical stimulation. $2,7,20$ The motor area was found to be immediately adjacent $(<1 \mathrm{~mm})$ to the SSS for a length of $40 \mathrm{~mm}$. This finding enables future work to determine the location of motor area by use of venography landmarks alone in this sheep model.

The anatomy of the cerebral venography of the sheep with regard to the motor areas is generalizable to humans. We recently reported the diameters of the human cerebral venous system and proximity to motor area. Unlike the human brain, the sheep brain is characterized by a motor strip that runs in an anterior projection, immediately adjacent to the SSS in the parasagittal plane. The human motor area runs in a lateral projection, with the CSV the corollary adjacent venous structure. The diameters of the sheep SSS measure between 1.2 and $2.4 \mathrm{~mm}$, while the human CSV measures between $2.3 \mathrm{~mm}$ and $4.9 \mathrm{~mm} .{ }^{15}$ The CSV may be the ideal target for a human endovascular brainmachine interface array, making the sheep SSS a suitable preclinical target.

Catheter venography including sinus structures is technically challenging due to irregular anatomy, including venous valves, webs, anatomical variability, and irregular junctions. ${ }^{23}$ Achieving distal cerebral vein access with a wide-bore catheter required use of a telescoping coaxial catheter delivery system. Stiffness of the distal catheters was an important consideration, with the larger, stiffer catheters more likely to cause dissection, and the softer catheters less likely to advance distally. A technique of proximal catheter support with forward pressure and twisting of the delivery catheter was found to be most effective at overcoming the most challenging segment (the torcular).

We assessed a range of commercially available catheters to determine the ideal coaxial system to safely navigate the convoluted venous pathway to the SSS. The increase in complications of dissection and hemorrhage appeared to be mostly due to the size and stiffness of the catheter. All complications related to catheters occurred with use of the 5-Fr and 6-Fr catheters, with no complications associated with the 2- and 4-Fr catheters. It may also be partly explained by a learning effect, with improvement in skill of the 3 operators (Oxley, Opie, Rind) occurring over the 2 years and 39 procedures. Attempted advancement of the larger catheters into the SSS occurred in the first year and ceased following the reported complications. The 4-Fr 0.044-inch DAC catheter had the highest rate of success of accessing the SSS (100\%) with no associated complications. With an internal lumen diameter of $1.1 \mathrm{~mm}$, this catheter size appears to be the largest wide-bore lumen catheter capable of being safely delivered to the SSS in the sheep model. We found that even use of a soft guidewire (0.035-inch Benston) was associated with venous perforation with little driving force. Venous walls are thinner than arterial walls and lack a substantial muscular wall. We reported no perforations with use of a microwire (0.014-inch Transend 14) employing a "J" curve to negotiate difficult bifurcations.

A limitation of the study includes the anatomical generalizability of the model. While the SSS in the sheep provides a suitably sized venous target to enable the development of a preclinical neural interface, the anatomical environment contains differences that require consideration. The SSS in a human is at least $6 \mathrm{~mm} .{ }^{15}$ While the diameter of the SSS in sheep provides a corollary to the superficial cortical veins in humans, the lack of a dural wall in the cortical veins in humans may represent a significant difference with consideration of stent deployment safety, vessel wall incorporation, and signal recording quality. Further work is required to demonstrate the safety of implantation into direct cortical veins. With regard to validation of motor prostheses models, a limitation of the sheep model is the inability to achieve complex motor training paradigms to investigate closed-loop algorithms. The final development of an endovascular neural interface for the purpose of brain-machine interface research may require transition into a primate model, but nonhuman primate research is becoming increasingly expensive and ethically questionable. Substantial work can be achieved in preclinical neural interface development, including validation and biocompatibility studies.

\section{Conclusions}

In this paper we report a novel ovine model for cerebral venography with generalizability to the human equivalent with respect to the location of the motor cortex. The challenges of tortuous venous catheterization can be overcome with a telescoping coaxial catheter system and specific surgical techniques. The ovine model may enable development and translation investigations of the novel field of endovascular neural interfaces. Applications include preclinical investigations for cortical recording technologies for paralysis and epilepsy, as well as other potential uses in neuromodulation that include the targeting of superficial and deep neural structures adjacent to blood vessels.

\section{Acknowledgments}

The research was supported by Defense Advanced Research Projects Agency (DARPA) MTO Contract No. N66001-12-14045; the Office of Naval Research (ONR) Global No. N6290914-1-N020; National Health and Medical Research Council of Australia (NHMRC) Project Grant APP1062532 and Development Grant APP1075117; Defence Health Foundation, Australia (Booster Grant); Defence Science Institute, Australia, grant; and Brain Foundation, Australia, research gift. Dr. Oxley acknowledges the support of the Royal Melbourne Hospital Neuroscience Foundation for the 
Warren Haynes Fellowship, as well as the Faculty of Medicine, University of Melbourne, for the Leslie Eric Paddle Scholarship in Neurology.

\section{References}

1. Antiga L, Piccinelli M, Botti L, Ene-Iordache B, Remuzzi A, Steinman DA: An image-based modeling framework for patient-specific computational hemodynamics. Med Biol Eng Comput 46:1097-1112, 2008

2. Bagley C: Cortical motor mechanism of the sheep brain. Arch Neurol Psychiatry 7:417-453, 1922

3. Barrese JC, Rao N, Paroo K, Triebwasser C, Vargas-Irwin C, Franquemont L, et al: Failure mode analysis of silicon-based intracortical microelectrode arrays in non-human primates. J Neural Eng 10:066014, 2013

4. Carmena JM, Lebedev MA, Crist RE, O’Doherty JE, Santucci DM, Dimitrov DF, et al: Learning to control a brainmachine interface for reaching and grasping by primates. PLoS Biol 1:E42, 2003

5. Chapin JK, Moxon KA, Markowitz RS, Nicolelis MAL: Real-time control of a robot arm using simultaneously recorded neurons in the motor cortex. Nat Neurosci 2:664-670, 1999

6. Fedorov A, Beichel R, Kalpathy-Cramer J, Finet J, FillionRobin JC, Pujol S, et al: 3D Slicer as an image computing platform for the Quantitative Imaging Network. Magn Reson Imaging 30:1323-1341, 2012

7. Grovum WL, Gonzalez JS: Electrical stimulation of the medial frontal lobe elicits a pattern of body movements in sheep. Brain Res 851:252-253, 1999

8. Hochberg LR, Bacher D, Jarosiewicz B, Masse NY, Simeral JD, Vogel J, et al: Reach and grasp by people with tetraplegia using a neurally controlled robotic arm. Nature 485:372375,2012

9. Hochberg LR, Serruya MD, Friehs GM, Mukand JA, Saleh M, Caplan AH, et al: Neuronal ensemble control of prosthetic devices by a human with tetraplegia. Nature 442:164-171, 2006

10. Hoffmann A, Stoffel MH, Nitzsche B, Lobsien D, Seeger J, Schneider H, et al: The ovine cerebral venous system: comparative anatomy, visualization, and implications for translational research. PLoS ONE 9:e92990, 2014

11. Lemon R: The output map of the primate motor cortex. Trends Neurosci 11:501-506, 1988

12. Liyanage KA, Steward C, Moffat BA, Opie NL, Rind GS, John SE, et al: Development and implementation of a Corriedale ovine brain atlas for use in atlas-based segmentation. PLoS One 11:e0155974, 2016

13. McConnell GC, Rees HD, Levey AI, Gutekunst CA, Gross RE, Bellamkonda RV: Implanted neural electrodes cause chronic, local inflammation that is correlated with local neurodegeneration. J Neural Eng 6:056003, 2009

14. Opie NL, John SE, Rind GS, Ronayne SM, Grayden DB, Burkitt AN, et al: Chronic impedance spectroscopy of an endovascular stent-electrode array. J Neural Eng 13:046020, 2016

15. Oxley TJ, Opie NL, John SE, Rind GS, Ronayne SM, Wheeler TL, et al: Minimally invasive endovascular stent-electrode array for high-fidelity, chronic recordings of cortical neural activity. Nat Biotechnol 34:320-327, 2016

16. Penfield W, Boldrey E: Somatic motor and sensory representation in the cerebral cortex of man as studied by electrical stimulation. Brain 60:389-443, 1937

17. Prasad A, Xue QS, Sankar V, Nishida T, Shaw G, Streit WJ, et al: Comprehensive characterization and failure modes of tungsten microwire arrays in chronic neural implants. J Neural Eng 9:056015, 2012

18. Santhanam G, Ryu SI, Yu BM, Afshar A, Shenoy KV:
A high-performance brain-computer interface. Nature 442:195-198, 2006

19. Serruya MD, Hatsopoulos NG, Paninski L, Fellows MR, Donoghue JP: Instant neural control of a movement signal. Nature 416:141-142, 2002

20. Simpson S, King JL: Localisation of the motor area in the sheep. Exp Physiol 4:53-65, 1911

21. Sun J, Wang J, Jie L, Wang H, Gong X: Visualization of the internal cerebral veins on MR phase-sensitive imaging: comparison with 3D gadolinium-enhanced MR venography and fast-spoiled gradient recalled imaging. AJNR Am J Neuroradiol 32:E191-E193, 2011

22. Taylor DM, Tillery SI, Schwartz AB: Direct cortical control of 3D neuroprosthetic devices. Science 296: 1829-1832, 2002

23. Tsutsumi S, Nakamura M, Tabuchi T, Yasumoto Y, Ito M: Venous lacunae presenting with unusual upward protrusion: an anatomic study using high-resolution magnetic resonance imaging. Childs Nerv Syst 29:465-468, 2013

24. Velliste M, Perel S, Spalding MC, Whitford AS, Schwartz AB: Cortical control of a prosthetic arm for self-feeding. Nature 453:1098-1101, 2008

25. Wang W, Collinger JL, Degenhart AD, Tyler-Kabara EC, Schwartz AB, Moran DW, et al: An electrocorticographic brain interface in an individual with tetraplegia. PLoS One 8:e55344, 2013

26. Yanagisawa T, Hirata M, Saitoh Y, Kishima H, Matsushita $\mathrm{K}$, Goto T, et al: Electrocorticographic control of a prosthetic arm in paralyzed patients. Ann Neurol 71:353-361, 2012

27. Yushkevich PA, Piven J, Hazlett HC, Smith RG, Ho S, Gee JC, et al: User-guided 3D active contour segmentation of anatomical structures: significantly improved efficiency and reliability. Neuroimage 31:1116-1128, 2006

\section{Disclosures}

Dr. Oxley has received support of non-study-related clinical or research effort from Synchron. Dr. Mitchell has received support of non-study-related clinical or research effort from Codman Johnson \& Johnson, Stryker, and Medtronic. Dr. Davis has received modest travel support from Boehringer Ingelheim, BMS, Pfizer, and Medtronic; and has received modest honoraria from Boehringer Ingelheim and Medtronic for serving on an advisory board. Drs. Oxley and Opie hold shares in SmartStent Pty, Ltd., and Synchron, Inc.

\section{Author Contributions}

Conception and design: Oxley, Opie, Rind, John, Ronayne, Grayden, Desmond, Davis, O'Brien, May. Acquisition of data: Oxley, Opie, Rind, Liyanage, John, Ronayne, McDonald, Dornom, Lovell, Warne, Steward, O’Brien, May. Analysis and interpretation of data: Oxley, Rind, Liyanage, John, Ronayne, Dornom, Lovell, Mitchell, Bennett, Baquier, Steward, Grayden, Desmond, O'Brien, May. Drafting the article: Oxley, Rind, O’Brien, May. Critically revising the article: Oxley, Davis, May. Reviewed submitted version of manuscript: Oxley. Approved the final version of the manuscript on behalf of all authors: Oxley. Statistical analysis: Oxley. Administrative/technical/material support: Oxley. Study supervision: Oxley.

\section{Correspondence}

Thomas J. Oxley, Vascular Bionics Laboratory, Melbourne Brain Centre, Departments of Medicine and Neurology, Royal Melbourne Hospital, The University of Melbourne, Parkville, Victoria 3050, Australia.email: thomas.oxley@unimelb.edu.au. 